\title{
Thermally Assisted Magnetic Recording using Plasmon Antenna with Ultra Short Laser Pulse
}

\author{
H. Mano, H. Yoshikawa, H. Hashiba*, Y. Ashizawa*, A. Tsukamoto*, Y. Sasaki**, \\ S. Saito**, M. Takahashi**, S. Ohnuki*, and K. Nakagawa* \\ Graduate School of Science and Technology, Nihon University, 7-24-1 Narashinodai, Funabashi 274-8501, Japan \\ *College of Science and Technology, Nihon University, 7-24-1 Narashinodai, Funabashi 274-8501, Japan \\ ${ }^{* *}$ Graduate School of Engineering, Tohoku University, 6-6-05 Aoba-yama, Sendai 980-8579, Japan
}

As one of the methods for ultra high density recording of hard disk drives, Thermally Assist Magnetic Recording (TAMR) increases memory density and thus attracts attention. The key technology of the TAMR is applying laser light as well as plasmon antenna to generate localized near-field spot. We fabricated plasmon antennas on magnetic film to understand fundamental recording capability by confined near-field stimulated by ultra short laser pulse. Some square plasmon gold antennas were deposited over a magnetic recording film $\left(\mathrm{C}_{55} \mathrm{Pt}_{30} \mathrm{Cr}_{15}-\mathrm{SiO}_{2}\right)$. We successfully confirmed that small magnetic domains of $67 \mathrm{~nm} \times 62 \mathrm{~nm}$ were written at the corner of the plasmon antennas by the surface plasmon effect with a femto-second laser pulse.

Key words: HAMR, TAMR, thermally assisted recording, magnetic recording, plasmon, near-field, femto-second

\section{Introduction}

Magnetic recording technology needs to be increased in memory density beyond several tera-bits per square inch, because the amount of digital data is increasing exponentially around the world. Thermally Assist Magnetic Recording (TAMR) is one of the methods to make a breakthrough for ultra high density recording of hard disk drive. This technique enables a high steady recording film to switch its magnetization by the temporary heat generated by laser light. The recording minimum domain size written by TAMR, however, is limited by the heat spot, which is determined by the diffraction limit. Plasmon antenna can generate a smaller heat spot beyond the diffraction limit by the localized surface plasmon effect. When we lead a laser light to the plasmon antenna close to magnetic core, as illustrated in Fig. 1, the plasmon antenna can create a small near-field spot over recording film. To study the process of TAMR, we directly fabricated plasmon antennas on a recording film $\left(\mathrm{C}_{55} \mathrm{Pt}_{30} \mathrm{Cr}_{15}-\mathrm{SiO}_{2}\right)$, as shown in Fig. 2, to confirm the plasmonic enhancing effect at the plasmon antennas by laser irradiation ${ }^{1)}$. We successfully observed written smaller magnetic domains compared with laser spot size, but some randomly written magnetic domains, independently located from plasmon antennas, were also observed, as shown in Fig. 2. The reason why some random domains are written must be that the plasmon intensity enhancing effect is not enough to thermally write.

In this study, we carefully designed a new square plasmon antenna to create higher enhancing plasmon effect as well as to confine smaller intensity spot than previous study. We also chose recording conditions considering the magnetic characteristics of recording film depending on temperature to avoid unnecessary random written domains.

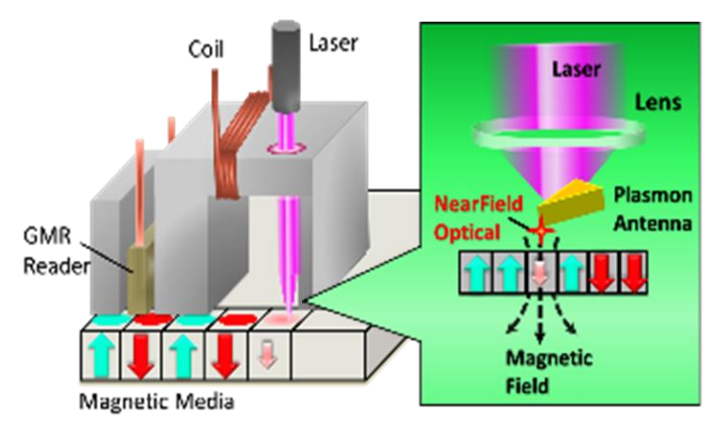

Fig. 1 Schematic view of Thermally Assisted Magnetic Recording (TAMR).

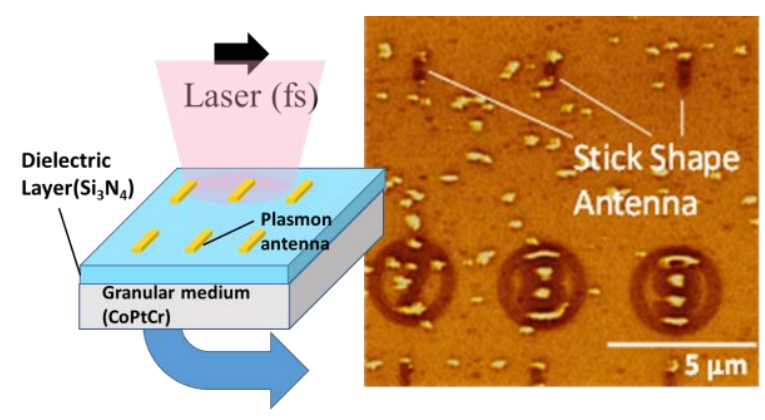

Fig. 2 Schematic diagram of the experiment and magnetic domains written by 90 fs laser pulse train ${ }^{1)}$

\section{Recording medium}

FePt magnetic films are studied as a film with high uniaxial magnetic anisotropy $K_{\mathrm{u}}$ for TAMR, but Curie temperature $T_{\mathrm{C}}$ of $\mathrm{FePt}\left(T_{\mathrm{C}} \sim 477{ }^{\circ} \mathrm{C}\right)^{2)}$ is too high to apply FePt to TAMR. Some studies reducing $T_{\mathrm{C}}$ and/or fabricating granular structure for FePt have been also investigated ${ }^{3-5}$, but they are still being studied. Then, we chose a $\mathrm{C}_{55} \mathrm{Pt}_{30} \mathrm{Cr}_{15}$ granular medium as a recording 
film to test possibility by the experiment of near-field recording, because it was already established in our group to fabricate $\mathrm{C}_{55} \mathrm{Pt}_{30} \mathrm{Cr}_{15}$ granular film which had an appropriate coercive force $H_{\mathrm{C}}$ and a low $T_{\mathrm{C}}{ }^{1,6)}$. The recording magnetic film ( $\mathrm{Co}_{55} \mathrm{Pt}_{30} \mathrm{Cr}_{15}$ granular film) fabricated on Si substrate by magnetron sputtering is $15 \mathrm{~nm}$ in total thickness with an under layer of $\mathrm{Ru}(20$ $\mathrm{nm}) / \mathrm{Pt}(6 \mathrm{~nm}) / \mathrm{Ta}(5 \mathrm{~nm})$, as shown in Fig. 3. The surface of the magnetic layer is coated by $\operatorname{SiN}(3 \mathrm{~nm})$ film to keep a distance between an antenna and the recording magnetic layer. Magnetic properties measured by polar Kerr effect from room temperature up to $290{ }^{\circ} \mathrm{C}$ are shown in Fig. 4. The saturation magnetic field $H_{\mathrm{S}}$ and the nucleation field $H_{\mathrm{N}}$ of the magnetic layer are more than $4 \mathrm{kOe}$ and less than $-1 \mathrm{kOe}$ at room temperature, respectively. Therefore, we can choose a magnetic bias field for recording between $-1 \mathrm{kOe}$ and 0 Oe without nucleation, and written domains can be kept at room temperature.

\section{Simulation analysis}

\subsection{Simulation model}

We propose a new square plasmon antenna as shown in Fig. 5 in order to enhance stronger plasmon effect than that of the previous stick antenna ${ }^{1)}$. As compared with the stick antenna, conduction electrons at the corner of the square antenna must be squeezed, and enhance the plasmon effect. The square Au antenna is located on a $\mathrm{Co}$ layer instead of $\mathrm{Co}_{55} \mathrm{Pt}_{30} \mathrm{Cr}_{15}-\mathrm{SiO}_{2}$ with $\mathrm{SiN}$ inter-layer on $\mathrm{SiO}_{2}$ substrate in the simulation model. Light source is linear polarized, $780 \mathrm{~nm}$ in wavelength, and $90 \mathrm{fs}$ pulse. The electric field direction of applied polarized light is set to the horizontal direction, as shown as an arrow in Fig. 5.

\subsection{Optical and Thermal Analyses}

Calculated intensity of electric field and temperature distribution are shown in Figs. 6 (a), and (b). As we expected, a high intensity local area is observed at the left- and right-corner of the square antenna, but at the top- nor bottom-corner, as shown in Fig. 6 (a). The calculated temperature distribution along with the horizontal direction after 90 fs laser irradiation, as shown in Fig. 6 (b), shows 5.82 times enhancing plasmon effect at the corner of the antenna. This enhancing effect is much higher than that of the previous stick shape antenna $(1.92 \text { times })^{1)}$.

\section{Fabrication of antenna on recording film}

We fabricated square antennas on the $\mathrm{C}_{55} \mathrm{Pt}_{30} \mathrm{Cr}_{15}$ $-\mathrm{SiO}_{2}$ recording film coated with $\mathrm{SiN}$ layer of $3 \mathrm{~nm}$ in thickness. The antennas are made of $\mathrm{Au}$, and $30 \mathrm{~nm}$ in thickness. The side length $L$, as defined in Fig. 5, is ranging from 100 to $1000 \mathrm{~nm}$. These antennas were fabricated by applying the E-beam lithographic technique as well as the lift-off method.

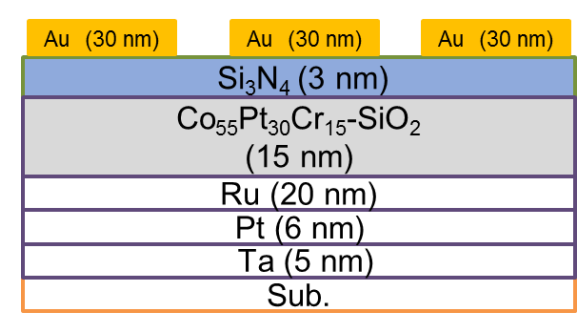

Fig. 3 Layer structure of recording medium with $\mathrm{Au}$ plasmon antenna ${ }^{1)}$.

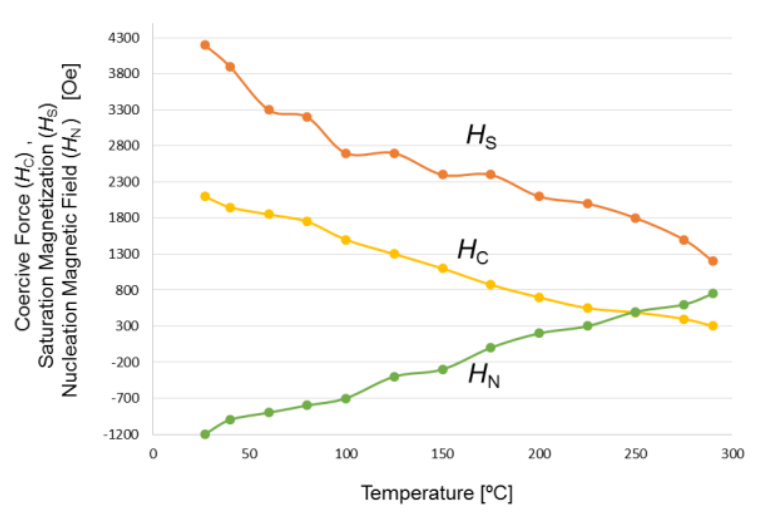

Fig. 4 Magnetic properties of coercive force $H_{\mathrm{C}}$, saturation field $H_{\mathrm{s}}$, and nucleation field $H_{\mathrm{N}}$ of a $\mathrm{Co}_{55} \mathrm{Pt}_{30} \mathrm{Cr}_{15}-\mathrm{SiO}_{2}$ recording film.

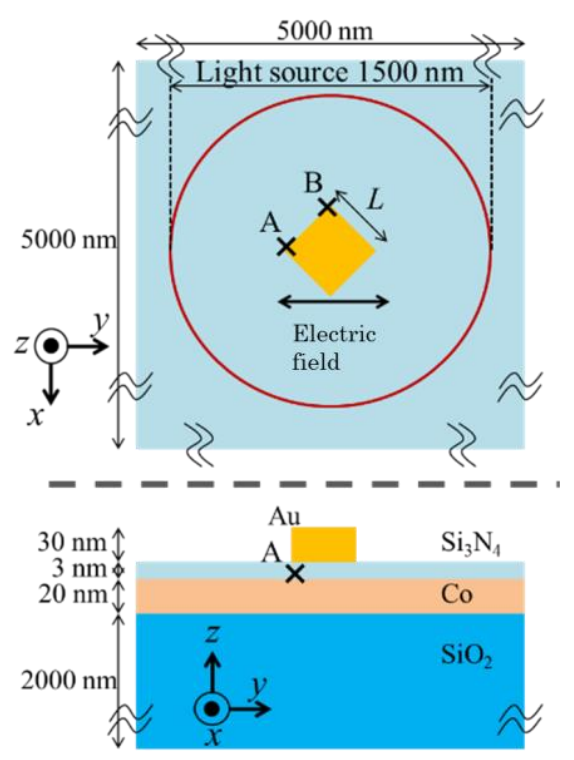

Fig. 5 The simulation model of electromagnetic field analysis and thermal diffusion analysis. 


\section{Thermally Assisted Magnetic Recording}

\subsection{Recording test without antenna}

First, the recording magnetic film was magnetized in the normal direction by applying magnetic field up to 10 kOe. Second, a bias magnetic field $H_{\mathrm{B}}$ of -300 Oe was applied. This condition of $\left|H_{\mathrm{B}}\right|<\left|H_{\mathrm{N}}\right|$ at room temperature, as shown in Fig. 4, can avoid coming out of domain nucleation. After rising temperature of the film under the condition of $\left|H_{\mathrm{C}}\right|<\left|H_{\mathrm{B}}\right|$ by laser heating, magnetization direction is reversed to follow to the direction of $H_{\mathrm{B}}$.

To know the threshold laser power to write magnetic domains by thermal recording, we exposed laser to the magnetic film under applying $H_{\mathrm{B}}=-300$ Oe. We found that the minimum laser power which is able to write a domain at the center of laser beam is $1.49 \mathrm{~mW}$, and that the maximum laser power which cannot write random domain is $0.77 \mathrm{~mW}$, under the condition that the irradiating laser beam at recording medium is $\sim 50 \mu \mathrm{m}$ in diameter, $800 \mathrm{~nm}$ in wavelength, $1 \mathrm{kHz}$ in repetition frequency, and $90 \mathrm{fs}$ pulse. This means that the threshold of laser power to write domains is between 0.77 and $1.49 \mathrm{~mW}$. As the experimental laser power is limited, we cannot choose a laser power between 0.77 and $1.49 \mathrm{~mW}$. The threshold of laser power not to write random domains is $0.77 \mathrm{~mW}$, so we chose $0.77 \mathrm{~mW}$ to write domains with the plasmon antennas. When we use $0.77 \mathrm{~mW}$ for a recording laser power, we need a plasmon antenna which has a higher efficiency than 1.94 times $(=1.49 \mathrm{~mW} / 0.77 \mathrm{~mW})$. As we reported in the simulation, the enhancing effect of the square antenna is 5.82 times, which is higher than the required condition. Therefore, we use this condition for our experimental study. Recording domain size can be estimated as follows. The background temperature line in Fig. 6 (b), which means the temperature without plasmon effect, corresponds to the writing power of 0.77 $\mathrm{mW}$. The expected minimum domain size must be the width at the 1.94 times higher temperature of the background line. According to the Fig. 6 (b), the minimum recording domain diameter is expected to be $38.4 \mathrm{~nm}$. On the other hand, the maximum domain must be written just above the background temperature. Then, we expect $103 \mathrm{~nm}$ as the maximum recording domain diameter.

\subsection{Result of thermal recording}

Written magnetic domains were observed by Magnetic Field Microscope (MFM) sensing stray field from magnetic film. The observed surface morphology and the magnetic domain are shown in Figs. 7 and 8 , respectively. The observed square antennas in Figs. 7 and 8 are the same antenna. The square bright area in Fig. 7 corresponds to the plasmon antenna. We cannot observe any structure as a bright area except the square antenna in Fig. 7. The electric field direction of incident laser beam is placed in horizontal direction as

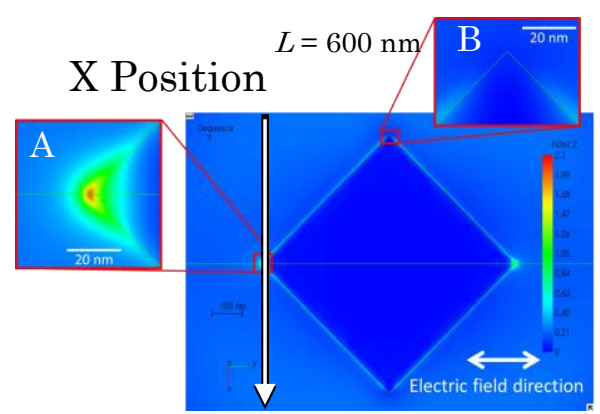

(a) Intensity of electric field

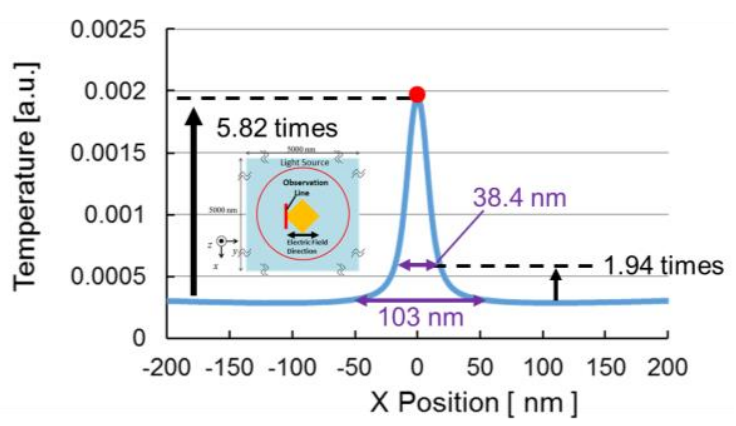

(b) Temperature distribution

Fig. 6 Calculated results for intensity of electric field (a) and temperature distribution (b).

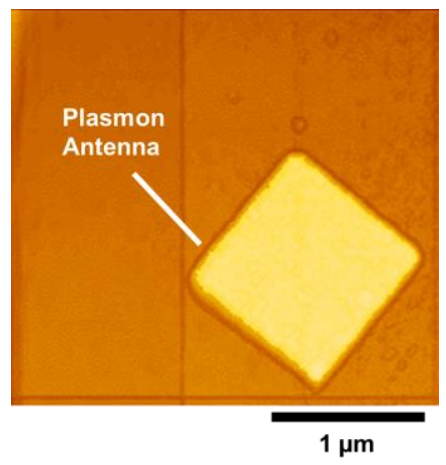

Fig. 7 Observation of the surface morphology.

shown as the arrows in Fig. 8. The square shape is also observed in the magnetic image, because an antenna thickness of $30 \mathrm{~nm}$ can affect magnetic field image caused by the feedback system in the MFM to keep distance between MFM prove and the surface of the sample. At the right hand side of the antenna in the MFM image in Fig. 8 (a), a bright part at the inside of the white circle can be seen, even though other bright areas are also observed on the right-bottom edge of the antenna. Such noise can appear when we observe samples at a steep physical step, because the MFM system has an automated keeping distance between the MFM prove and the 
sample, as mentioned before. The noise depends on the scanning direction. In Fig. 8 (a), the MFM image was measured with scanning from top to bottom. Therefore we observed the image with scanning from left to right, as shown in Fig. 8 (b). The only bright area which is shown in the circle in Fig. 8 (a) can be seen both Figs. 8 (a) and (b) independently scanning direction, so this bright part must be written magnetic domain by the effect of surface plasmon. Besides, we cannot observe any other random domains at the area without the antenna such as seen in Fig. 2 with stick antennas. This is the evidence that the surface plasmon effect can enhance the exposed laser light. The minimum domain, which indicates the size of $67 \mathrm{~nm} \times 62 \mathrm{~nm}$, is shown in Fig. 9. Comparing to previous experiments with the stick antenna, a minimum written domain size is reduced from $166 \mathrm{~nm} \times 120 \mathrm{~nm}$ to $67 \mathrm{~nm} \times 62 \mathrm{~nm}$. This achievement is caused by the sharp temperature distribution affected by surface plasmon effect at the corner of the square antenna.

\section{Conclusion}

We fabricated square antennas on a magnetic recording film for testing a local heating activity by near-field technique for TAMR. After $90 \mathrm{fs}$ laser pulses were irradiated over the plasmon antennas, small magnetic domains such as $67 \mathrm{~nm} \times 62 \mathrm{~nm}$ in size were observed by MFM at corners of the plasmon square antennas. This study revealed that the TAMR using near-field effect by plasmon antenna was effective to achieve future high density magnetic memory.

Acknowledgements This work is partially supported by a Grant-in-Aid for Scientific Research (C) \#23560413, a Grant of Storage Research Consortium, and a Grant of MEXT- Supported Program for the Strategic Research Foundation at Private Universities, 2013-2017.

\section{References}

1) A. Tajiri, K. Tamura, S. Toriumi, Y. Ashizawa, K. Nakagawa, A. Tsukamoto, A. Itoh, Y. Sasaki, S. Saito, M. Takahashi, and S. Ohnuki: J. Magn. Soc. Jpn., 37, 119-122 (2013).

2) D. Weller, A. Moser, L. Folks, M. E. Best, W. Lee, M. F. Toney, M. Schwickert, J-U. Thiele, and M. F. Doerner: IEEE Trans. Magn., 36, 10-15 (2000).

3) D. A. Gilbert, L. W. Wang, T. Klemmer, J. Thiele, C. Lai, and K. Liu: Appl. Phys. Lett, 102, 132406 (2013).

4) J. Tsukioka, T. Ubana, A. Tsukamoto, and A. Itoh: J. Magn. Soc. Jpn., 38, 143-146 (2014).

5) L. Zhang, Y. K. Takahashi, K. Hono, B. C. Stipe, J. -Y. Juang, and M. Grobis: IEEE Trans. Magn., 47, 4062-4065 (2011).

6) K. Nakagawa, Y. Osa, A. Tajiri, Y. Ashizawa, S. Ohnuki, Y. Sasaki, S. Saito, M. Takahashi, and A. Itoh: J. Magn. Soc. Jpn., 36, 58-61 (2012).

Received Dec. 25, 2014; Revised Feb. 28, 2015; Accepted Apr. 09, 2015

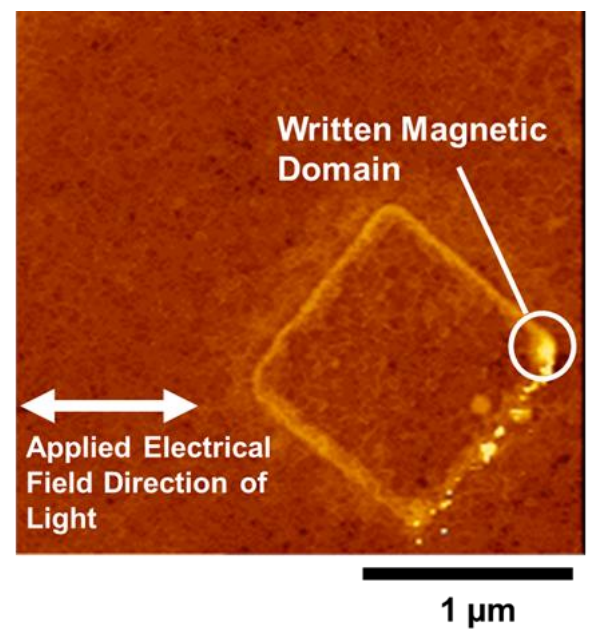

(a) Scanning direction of up to down.

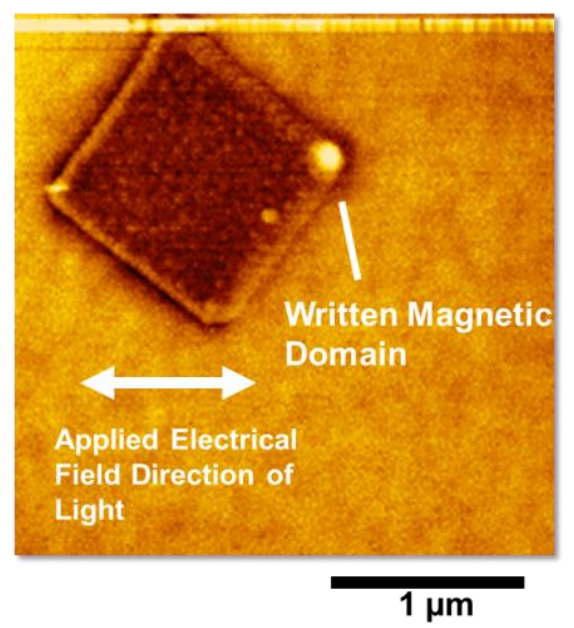

(b) Scanning direction of left to right.

Fig. 8 Observation of the magnetic domain after thermal recording. Scanning direction of (a) is up to down, scanning direction of (b) is left to right.

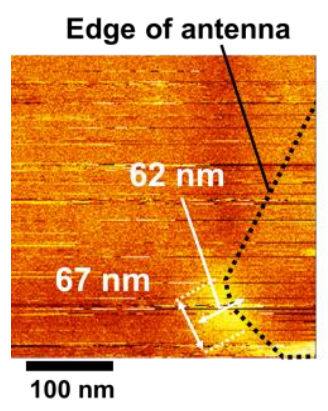

Fig. 9 Magnetic image of the minimum recorded domain. 